\title{
Longitudinally Stratified Combustion in Wave Rotors
}

\author{
M. Razi Nalim* \\ Indiana University-Purdue University at Indianapolis, Indianapolis, Indiana 46202
}

\begin{abstract}
A wave rotor may be used as a pressure-gain combustor, effecting wave compression and expansion, and intermittent confined combustion, to enhance gas-turbine engine performance. It will be more compact than an equivalent pressure-exchange wave-rotor system, but will have similar thermodynamic and mechanical characteristics. Because the allowable turbine blade temperature limits overall fuel-air ratio to subflammable values, premixed stratification techniques are necessary to burn hydrocarbon fuels in small engines with compressor discharge temperatures well below autoignition conditions. One-dimensional, nonsteady numerical simulations of stratified-charge combustion are performed using an eddy-diffusivity turbulence model and a simple reaction model incorporating a ftammability limit temperature. For good combustion efficiency, a stratification strategy is developed that concentrates fuel at the leading and trailing edges of the inlet port. Rotor and exhaust temperature profiles and performance predictions are presented at three representative operating conditions of the engine: full design load, $40 \%$ load, and idle. The results indicate that peak local gas temperatures will cause excessive temperatures in the rotor housing unless additional cooling methods are used. The rotor temperature will be acceptable, but the pattern factor presented to the turbine may be of concern, depending on exhaust duct design and duct-rotor interaction.
\end{abstract}

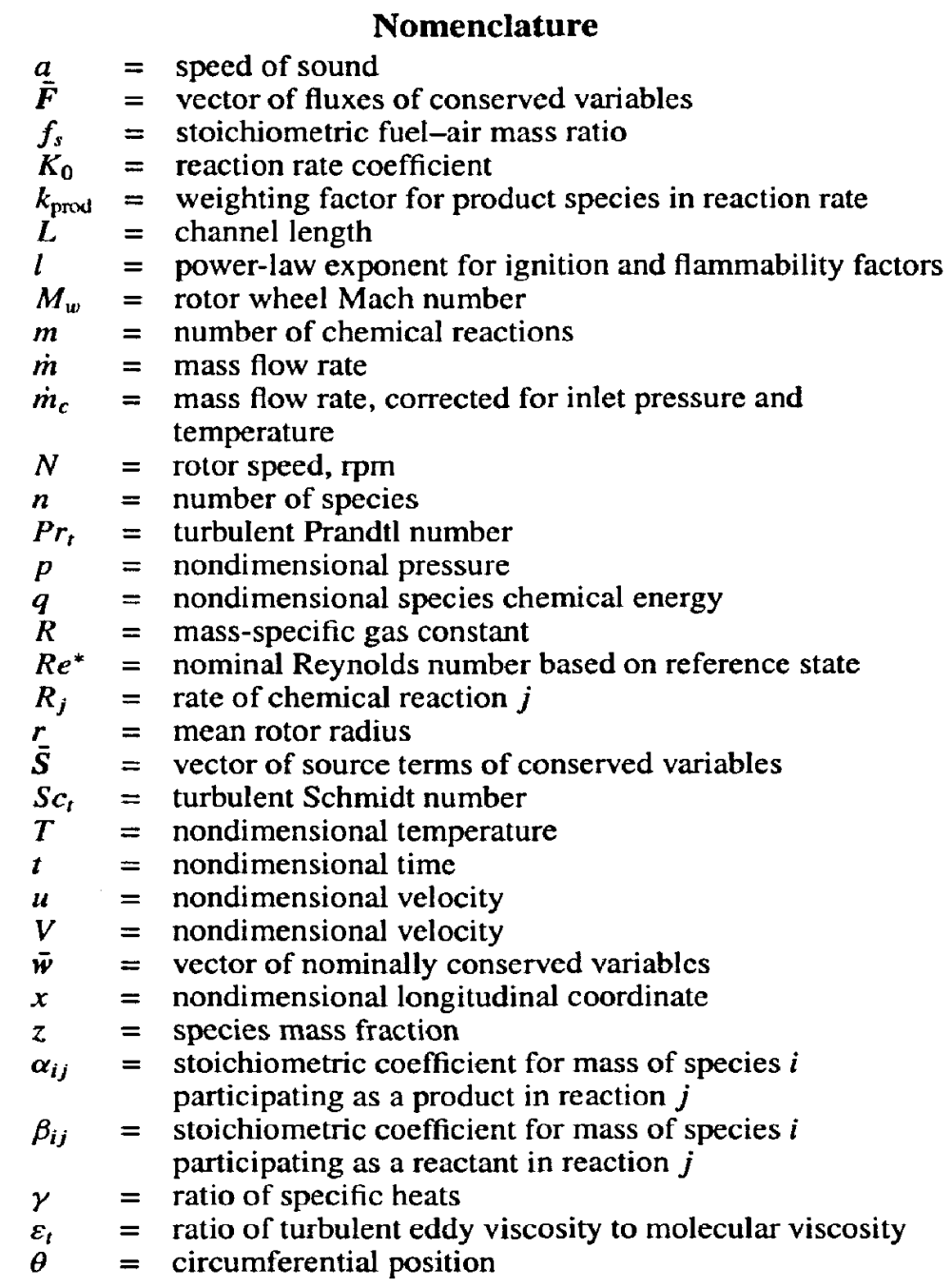

Received 23 August 1999; accepted for publication 9 December 1999. Copyright (C) 2000 by M. Razi Nalim. Published by the American Institute of Aeronautics and Astronautics, Inc., with permission.

*Assistant Professor, Department of Mechanical Engineering, 723 W. Michigan Street, SL260. Senior Member AIAA.
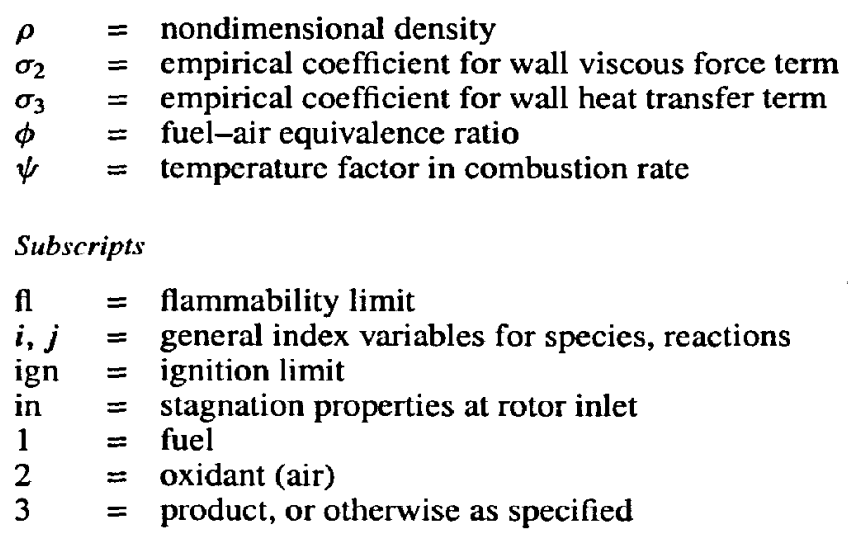

Superscript

* $\quad=$ reference state property (dimensional quantity), when applied to symbol for corresponding nondimensional quantity

\section{Introduction}

WAVE rotor is a device that utilizes nonsteady wave motion to
exchange energy by direct work action between fluids, which
may be chemically inert or reacting. It consists of a number of chan-
nels arranged about an axis. By rotation, the ends of the channels are
periodically ported to high- and low-pressure manifolds (ducts) that
synchronically generate and utilize waves in the channels. Because
the number of channels is large, the flow in the ducts is practically
steady and is directed to other steady flow components. An im-
portant feature is that as gases of a wide temperature range flow
through the rotor, the mean temperature of the channel wall is lower
than the highest gas temperature. Rotational speed is low relative to
conventional turbomachines, and the geometry is usually simpler,
allowing greater strength and lower cost. For detailed descriptions
of wave-rotor principles and applications see Refs. 1-3.
A wave rotor acting as a pressure exchanger can be used (together
with a conventional combustor) as a topping unit to enhance the per-
formance of a gas-turbine engine. Fundamental thermodynamics
and detailed simulations ${ }^{1,5}, 6$ based on validated codes indicate a
substantial pressure gain possible between the compressor and the
turbine. Alternatively, this pressure gain could be obtained using
an internal-combustion wave rotor. In this case, combustion occurs 


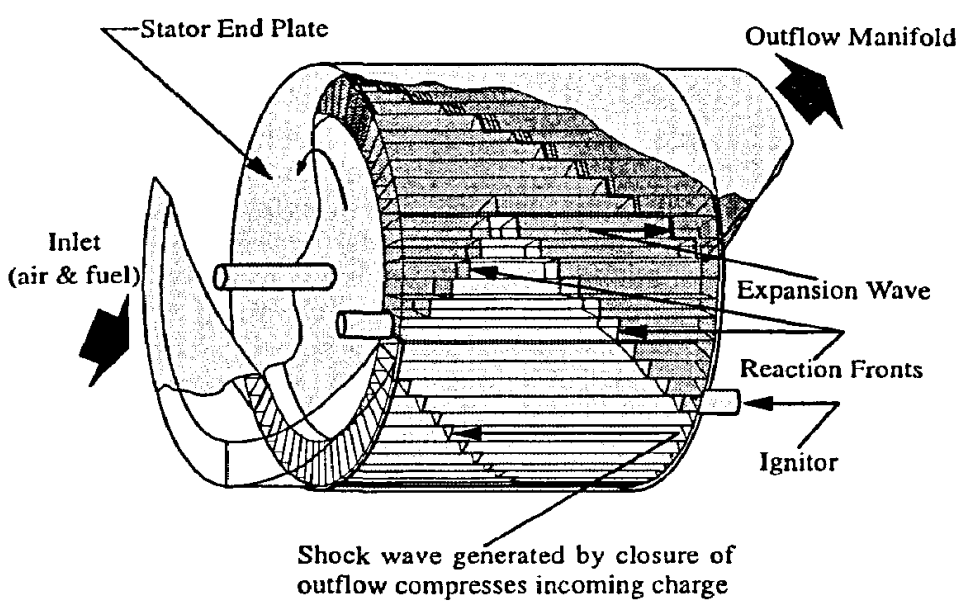

Fig. 1 Internal-combustion wave-rotor schematic. ${ }^{8}$

sequentially within the wave channels, each channel being periodically charged and discharged as it rotates past properly sized and timed inlet and outlet ports. Simplified combustion and wave processes are illustrated in the wave-rotor sketch in Fig. 1, taken from Ref. 7. By accomplishing combustion in the rotor, the external combustor needed in a pressure-exchanger topping cycle is eliminated, as is the associated ducting, which may be long and unmanageably hot. The importance of thermal issues in any wave rotor for gas-turbine enhancement was anticipated, but this work reveals that the cause and location of thermal loads are not obvious.

\section{Objectives}

To minimize rotor size and losses, a rapid and reliable mode of combustion is desired. For small engines with relatively low inlet temperature $(<800 \mathrm{~K})$, a premixed turbulent flame-propagation mode is envisaged. ${ }^{8}$ With higher air temperature, autoignited modes (detonation or nonpremixed combustion) become possible. In a previous study, ${ }^{7}$ deflagrative and detonative premixed modes were simulated using a one-dimensional computational fluid dynamics (CFD) code with a simple combustion model. Single design-point computations were performed assuming uniform inlet mixtures with the desired fuel energy content, without consideration of the flammability limits of real fuels over the operating range of the engine. That simplification was necessitated by the use of a single reaction progress variable to model heat release.

This paper reports a generalization of the earlier model to represent multiple species and reaction steps, and the incorporation of a flammability-limited combustion model to simulate combustor operation over a wide range. Flammability limitations dictate mixture distribution in a wave rotor, which strongly influences thermal loads, as in other combustion engines. The present application is limited to premixed turbulent flames ignited by residual and reinjected hot gas, with single-step reaction. Specifically, the model is used to examine the possibility of operating a small turboshaft engine $(2.2 \mathrm{~kg} / \mathrm{s})$ near sea level using pressure-gain, wave-rotor combustion. Other limitations of one-dimensional simulations and simplified combustion and turbulence models discussed by Nalim and Paxson ${ }^{7}$ still apply. Available data on flammability limits and flame propagation rates are used to estimate the wave-rotor design parameters.

Under all operating conditions of a gas-turbine engine the overall fuel-air ratio is usually below the lean flammability limit of typical fuels. This necessitates the use of a richer primary zone for stable combustion in conventional combustors, with additional air being mixed in downstream to bring the overall mixture to turbineacceptable temperature. In wave-rotor combustion, a similar strategy is necessary. It consists of separating the inlet air into a number of premixed sectors fueled by separate continuous-flow fuel injectors, enriching sectors that supply the zones where combustion is initiated. As load varies, injectors are modulated to maintain flammable mixture or are turned off. This may require electronic control of fuel flow, although none more complex than are used on modern engines. Simulation of the combustion of such stratified mixtures required the present model.

The internal-combustion wave-rotor model will be presented first, consisting of governing equations and the method of solution. The design of a wave rotor for the specific engine application is then discussed, and simulations are presented for full design load, part load, and idle conditions of operation of the engine. Feasibility issues and foreseen design challenges are explored in conclusion.

\section{Computational Model and Solution Method}

The computational model consists of a representative wave-rotor channel interacting with the upstream compressor, downstream turbine, channel walls, leakage cavity, and hot-gas reinjection passage (Fig. 2), as in Ref. 7. The leakage cavity represents space within the rotor housing that can exchange gas with the channels via the end gaps between the rotor and the casing endwalls. The reinjection passage takes hot combustion gas from channels at high pressure for ignition of fresh mixture in other channels. The total pressure and temperature delivered by the compressor is specified at each operating condition and no compressor model is used. The turbine is modeled as a choked valve with a fixed area, which produces a back pressure matched to the flow rate. The channel walls are divided into axial elements matching the channel computational cells. Each element exchanges heat with the corresponding fluid cell and the leakage cavity, but not with adjacent segments; that is, longitudinal conduction is neglected.

\section{Channel Governing Equations}

Extension of the model to multiple species allows the use of nonuniform mixtures in the simulation. Calorically perfect gas is assumed, allowing the use of a constant (average) specific heat and gas constant, irrespective of local composition. The governing equations for an arbitrary number of $n$ species and $m$ reactions are given hereafter, consisting of total mass, momentum, and energy conservation equations, and $(n-1)$ equations for masses of all species but one. A species may be a single chemical compound, or a substance (such as air) that can be accounted for as a combination of compounds in fixed proportions, so that fewer equations need to be solved.

In nondimensional form the equations are

$$
\frac{\partial \bar{w}}{\partial t}+\frac{\partial \bar{F}(\bar{w})}{\partial x}=\bar{S}(\bar{w})
$$

where

$$
\vec{w}=\left[\begin{array}{c}
\rho \\
\rho u \\
\frac{p}{\gamma(\gamma-1)}+\frac{\rho u^{2}}{2}+\sum_{i=1}^{n} \rho z_{i} q_{i} \\
\rho z_{i} \quad(i=1, n-1)
\end{array}\right]
$$

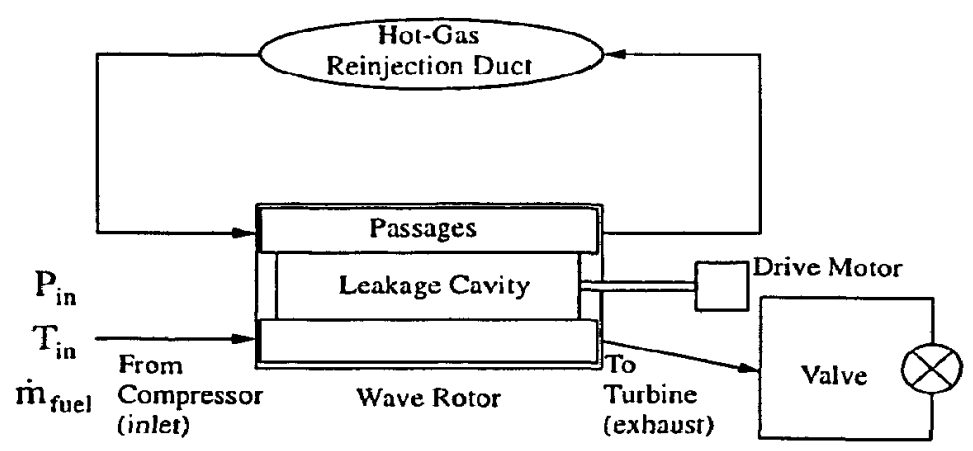

Fig. 2 System simulation schematic. 
and

$$
\overline{\boldsymbol{F}}=\left[\begin{array}{c}
\rho u \\
\frac{p}{\gamma}+\rho u^{2} \\
u\left(\frac{p}{\gamma-1}+\frac{\rho u^{2}}{2}+\sum_{i=1}^{n} \rho z_{i} q_{i}\right) \\
\rho u z_{i} \quad(i=1, n-1)
\end{array}\right]
$$

All gas properties have been normalized by their respective values at a single, arbitrarily chosen reference state, and the axial velocity $u$ has been normalized by $a^{*}$, the sound speed, at the reference state. The longitudinal coordinate $x$ has been normalized by the channel length, and time $t$ by the characteristic wave transit time $L / a^{*}$. The source vector given and described subsequently contains contributions from the chemical reactions, turbulent eddy diffusion, viscous forces, and heat transfer at the walls:

$$
\bar{S}(\bar{w}, x)=\left[\begin{array}{c}
0 \\
\frac{\varepsilon_{t}}{R e^{*}} \frac{\partial^{2} u}{\partial x^{2}}+\sigma_{2} u|\rho u|^{0.75} \\
\frac{\varepsilon_{t}}{R e^{*}} \frac{\partial^{2}}{\partial x^{2}}\left(\frac{u^{2}}{2}+\frac{T}{(\gamma-1) P r_{t}}+\sum_{i=1}^{n} \frac{z_{i} q_{i}}{S c_{t}}\right) \\
+\sigma_{3} u|\rho u|^{0.75}\left(T-T_{\text {watt }}\right) \\
\frac{\varepsilon_{t}}{R e^{*} S c_{t}} \frac{\partial^{2} z_{i}}{\partial x^{2}}+\sum_{j=1}^{m}\left(\alpha_{i j}-\beta_{i j}\right) R_{j} \\
(i=1, n-1)
\end{array}\right]
$$

A leakage term added only for the wall-adjacent gas is omitted here for clarity. The treatment of leakage, boundary conditions, heat transfer, and viscous losses follows the one-dimensional, finitechannel-width, wave-rotor calculations described by Paxson," with modifications, model coefficients, and extension to include turbulent mixing effects given in Ref. 8. The particular combustion model and reaction rate $R_{j}\left(T, p, z_{i}\right)$ used in this work are described next.

\section{Combustion Model and Reaction Rate}

In the calculations presented here, combustion in the wave rotor is modeled by a single reaction step involving three species: fuel, oxidant (air), and product. This is the simplest representation that allows for variable fuel-air ratio. The following discussion is a preamble to the formulation of an appropriate reaction rate.

In a finite volume computation, the thin moving reaction front, associated with the typically large activation energy of combustion reactions, cannot be easily resolved while using a uniform grid. Similarly, no account can be made of turbulence effects on scales smaller than the computational cell size and time step. Therefore, the reaction rates computed using cell-averaged variables must be assigned phenomenologically, based on a combination of chemical and turbulence effects. Turbulence effects are partly represented by the simple eddy diffusivity model.

The chemical kinetics of combustion can be modeled by choosing the reactions and species to consider. However, even when a large full set of chemical steps is used, the existence of flammability limits of reactant species concentrations cannot be accounted for without considering the physical processes that operate at extinction. ${ }^{10}$ The lean flammability limit has been accounted for when a heat-loss model is added to a full kinetic calculation; this is still an area of active and somewhat speculative research. Interestingly, the lean limit is not observed to be sensitive to the amount of heat loss. ${ }^{11}$ It appears to be related to a minimum energy content of reactants, so that the temperature of combustion products at the lean flammability limit is roughly a constant over a range of initial temperature of the mixture. This limit temperature is in the range of $1400-1600 \mathrm{~K}$ for hydrocarbon-air combustion. It allows for a simple total-energybased flammability limit to be incorporated in the present model.
The modeled reaction rate is determined by the minimum of three rates, each based on the concentration of one species, similar to Ref. 12. This implies that combustion will occur by a mixingcontrolled reaction at a propagating flame front at which all three species are present, with the most deficient species controlling the rate. The product species is given greater weight to account for its dominant role in providing active radicals (besides heat) for initiating reaction in the fuel-air mixture, which is initially well below autoignition temperature. Fuel and air are weighted according to stoichiometry. Large activation energy is assumed, so that the classical Arrhenius model becomes an ignition-temperature model, that is, the rate is zero below a threshold (ignition) temperature $T_{\mathrm{ign}}$. Finite volume computations based on a pure step function of this kind were found to be somewhat unstable, magnifying small variations in local conditions. Therefore, the rate is modeled to better simulate Arrhenius temperature dependence above the ignition temperature, by using a factor $\psi_{\text {ign }}$ with a power-law dependence on the excess temperature that asymptotically tends to unity at high temperature.

To account for the fall in reaction rate close to the lean flammability limit and extinction below it, another similar factor and a corresponding limit temperature $T_{\mathrm{ff}}$ is used. This factor introduces a rate dependence on the extended static temperature, $T_{e}=$ $T+(\gamma-1) z_{1} q_{1}$, which is the potential static temperature of the gas mixture after complete reaction and, therefore, measures the total thermal and chemical energy of the mixture.

The rate of the combustion reaction (consumption rate of fuel mass), incorporating the aforementioned features, is of the form

$$
\begin{aligned}
R_{1}= & K_{0} \rho\left\{\begin{array}{c}
1-\left(T_{\mathrm{ign}} / T\right)^{l_{\mathrm{gn}}} ; T>T_{\mathrm{ign}} \\
0 ; T<T_{\mathrm{ign}}
\end{array}\right\}\left\{\begin{array}{c}
1-\left(T_{\mathrm{f}} / T_{e}\right)^{l_{\mathrm{fi}}} ; T_{e}>T_{\mathrm{f}} \\
0 ; T_{e}<T_{\mathrm{fl}}
\end{array}\right\} \\
& \times \frac{1}{k_{\mathrm{prod}}} \min \left\{\begin{array}{c}
z_{1} \\
z_{2} f_{s} \\
k_{\mathrm{prod}} z_{3} /\left[1+\left(1 / f_{s}\right)\right]
\end{array}\right\}
\end{aligned}
$$

The stoichiometric coefficients for the three species are as folIows: fuel, $\alpha_{11}=0, \beta_{11}=1$; air, $\alpha_{21}=0, \beta_{21}=1 / f_{s}$; and product, $\alpha_{31}=1+1 / f_{s}, \beta_{31}=0$. The rate coefficient $K_{0}$ is to be specified based on the controlling turbulence timescale. For the ignition and flammability factors, values of exponents $l_{\mathrm{ign}}$ and $l_{\mathrm{f}}$ in the range of 2-3 were found to work well. Figure 3 is a graph of the ignition temperature factor $\psi_{\text {ign }}$ using such values.

\section{Numerical Method for Channel Flow and System}

The model numerically integrates the equations of motion in a single channel by the same method as described in Ref. 7 and will not be discussed in detail here. In essence, it is a high-resolution, second-order scheme based on the approximate Riemann solver of Roe, ${ }^{13}$ with flux-limited dissipation. Grid-independent solutions were achieved with 200 computational cells in a channel length.

The channel-flow equations are coupled to lumped-capacitance models of the channel walls, leakage cavity, hot-gas-reinjection loop, and the downstream valved cavity representing the turbine.

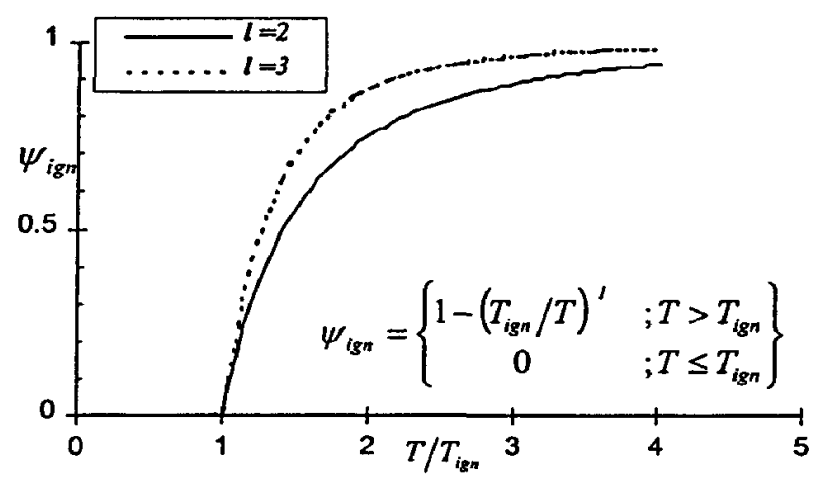

Fig. 3 Reaction rate temperature factor. 
Table 1 Wave-rotor dimensions and parameters

\begin{tabular}{lc}
\hline \hline Parameter & Value \\
\hline Mean rotor radius $r, \mathrm{~cm}$ (in.) & $8.15(3.2)$ \\
Rotor length $L, \mathrm{~cm}$ (in.) & $15.24(6.0)$ \\
Rotor channel height, cm (in.) & $2.18(0.86)$ \\
Design rotational speed (rpm) & 16,800 \\
Cycles/revolution & 2 \\
Number of channels & 52 \\
Inlet area, $\mathrm{cm}^{2}$ (in. $^{2}$ ) & $26(4.0)$ \\
\hline \hline
\end{tabular}

Because the time constants associated with transients in these objects are much longer than a complete wave cycle, their properties are updated once every cycle, until convergence to a steady state and a periodic channel-flow solution is obtained. Further details are provided in Ref. 7.

\section{Engine Design and Operating Range}

The simulations presented are for a pressure-gain wave rotor designed for a small engine with an upstream compressor pressure ratio of approximately 8.0 , as was done in the previous study. ${ }^{7}$ The major design parameters of this rotor are listed in Table 1. Equivalent external-combustion pressure-exchanger designs are reported in Refs. 5 and 14, which present performance predictions over a range of engine operating conditions. These studies predict that for a wave-rotor-topped engine in the $300-450 \mathrm{~kW}(400-600 \mathrm{hp}$ ) class, specific fuel consumption (SFC) and specific power can be enhanced significantly over the entire operating range. At the design point, the wave rotor produces a pressure gain of about $20 \%$ at the turbine inlet relative to the compressor discharge. This results in a specific power gain of $21 \%$ and SFC reduction of $17 \%$ over the baseline engine, similar to earlier predictions. ${ }^{1,6}$ Because of the self-cooling action of alternating hot and cold flow, the rotor is no hotter than the turbine and can use conventional materials.

In principle, a wave rotor with internal combustion will produce identical performance to a pressure-exchange wave rotor, if the inlet conditions from the compressor and exit conditions to the turbine are duplicated. However, combustion requirements will determine the size and speed of the rotor and, thus, its feasibility. In Ref. 7, the requirement of completing combustion in a reasonable time was met with average turbulent flame speeds of about $10 \mathrm{~m} / \mathrm{s}$ at the design point, assuming immediate ignition of each charge by reinjected hot gas and by residual hot gas. In this study, stratification of the charge into richer and shorter zones will result in faster combustion or allow lower turbulence intensity.

In a pressure-gain wave rotor discharging only to a high-pressure turbine, the channels cannot be completely purged with fresh cool air in each cycle because of the net adverse pressure difference across the wave rotor. For maximum self-cooling, the combustion process should be designed to occur as late as possible before exhaust, allowing for some cooler unburned gas to travel nearly the full length of the channels. ${ }^{7}$ Even then, the rotor wall will likely reach higher temperatures than an equivalent through-flow pressure exchanger. It will be revealed later that direct heat transfer to the channel walls is not the major thermal load in a combustion wave rotor.

Three operating points are considered in this study: $100 \%$ design load, $40 \%$ load, and idle. The pertinent conditions for these three points are listed in Table 2 . They are based on the pressure exchanger designs of Refs. 5 and 14 and the fuel properties assumed in the next section.

\section{Simulations}

The reference state for nondimensionalization of all variables at all conditions is the stagnation state of the compressor discharge (inlet to wave rotor) at the design conditions ( $p^{*}=7.6 \mathrm{~atm}$, $\left.T^{*}=600 \mathrm{~K}, a^{*}=485 \mathrm{~m} / \mathrm{s}\right)$. The simulations assume constant gas properties: $\gamma=1.353, R=290 \mathrm{~J} / \mathrm{kg} / \mathrm{K}$, lower heating value (LHV) of fuel $=40 \mathrm{MJ} / \mathrm{kg}, 1 / f_{s}=15$, and viscosity corresponding to $R e^{*}=$ $8.3 \times 10^{6}$, based on $a^{*}$ and $L$. For convenience, the chemical energy
Table 2 Topped-engine nominal operating conditions

\begin{tabular}{lccc}
\hline \hline Load & Design & $40 \%$ & Idle \\
\hline Power, kW & 520 & 220 & 0 \\
Mass flow, kg/s & 2.2 & 1.65 & 0.9 \\
Base pressure ratio & 7.6 & 5.4 & 2.5 \\
Heat rate, kW & 1800 & 1030 & 300 \\
Compressor exit temperature, $\mathrm{K}$ & 600 & 540 & 420 \\
Overall fuel-air ratio & 0.02 & 0.015 & 0.008 \\
Turbine inlet temperature, $\mathrm{K}$ & 1330 & 1100 & 700 \\
\hline \hline
\end{tabular}

Table 3 Turbulence and combustion parameters

\begin{tabular}{lc}
\hline Parameter & Value \\
\hline Eddy/molecular viscosity ratio $\varepsilon_{t}$ & 1000 \\
Turbulent Prandtl number $P r_{t}$ & 1.0 \\
Turbulent Schmidt number $S c_{t}$ & 1.0 \\
Rate coefficient $K_{0}$ & 75.0 \\
Ignition temperature $T_{\mathrm{ign}} T^{*}, \mathrm{~K}$ & 780.0 \\
Ignition temperature exponent $l_{\mathrm{ign}}$ & 2.0 \\
Flammability limit temperature $T_{\mathrm{ft}} T^{*}, \mathrm{~K}$ & 1560 \\
Flammability limit exponent $l_{\mathrm{fl}}$ & 3.0 \\
Product weighting factor $k_{\text {prod }}$ & 0.1 \\
\hline \hline
\end{tabular}

of the fuel is assigned the LHV: $q_{1}=170.0$, non-dimensionalized by $a^{* 2}$. The air and product species are, therefore, both at the zeroreference chemical energy level. Note that in a partially or fully combusted mixture, the air species consists of all remaining oxygen and accompanying nitrogen in the same proportion as fresh air, and the product species consists of reacted compounds and nitrogen in proportion to the consumed oxygen.

The turbulence and combustion rate parameters are kept fixed at the values given in Table 3 for all operating conditions. The values of these parameters will not be justified a priori. They are chosen to be within the range of turbulence levels and scales, and flame propagation rates, commonly observed in gas turbines and reciprocating engines utilizing hydrocarbon fuels. Nevertheless, combustion in wave rotors has unique features ${ }^{7,8}$ for which little prior experience exists. These include flame distortion and likely acceleration by strong pressure waves, rotational effects, and proximate walls at relatively high temperature. Future wave-rotor combustion experimental studies could be designed to calibrate the model presented by refining these parameter values. The choices of $\varepsilon_{t}$ and $K_{0}$ result in apparent flame speeds that are consistent with turbulence intensity corresponding to about $1 \%$ of the kinetic energy in the inlet flow. The prescribed flammability limit temperature corresponds to a limit equivalence ratio of 0.54 at room temperature $(300 \mathrm{~K})$, which will automatically shift to a lower equivalence ratio at higher temperature $(0.34$ at $750 \mathrm{~K})$.

The stratification technique is illustrated in Fig. 4, in wave diagrams of the flowfield for one cycle (half a revolution) of the wave rotor. These diagrams may be viewed, moving vertically upwards, as time traces of the flow properties in any one channel through one cycle. Port locations are given by circumferential position $\theta$ varying from 0 to $\pi$. From computations discussed hereafter, Fig. $4 a$ has a contour-line diagram of the pressure waves (isobars), superimposed over a gray-scale, contour-shade plot of the temperature (isotherms), and Fig. $4 \mathrm{~b}$ has a contour diagram of the fuel fraction with overlaid line and gray-scale plots. Lighter shades represent higher temperatures in Fig. 4a and higher fuel concentrations in Fig. 4b. Observe the cyclic continuity of the variables in this converged periodic solution, from the top edge to the bottom edge of each diagram. Quantitative information about this and other simulations is presented later.

Stratification of the premixed charge is arranged so as to ensure nearly complete combustion in all cases. The air inlet duct is divided into five equal circumferential sectors for injecting and mixing fuel, each about as wide as one rotor channel, and the mixture strength is specified in each sector. Sector 1 supplies the first layer of gas to the channel, which relies on residual hot gas for ignition. Sector 5 supplies the terminal, wall-adjacent layer, which must be ignited 


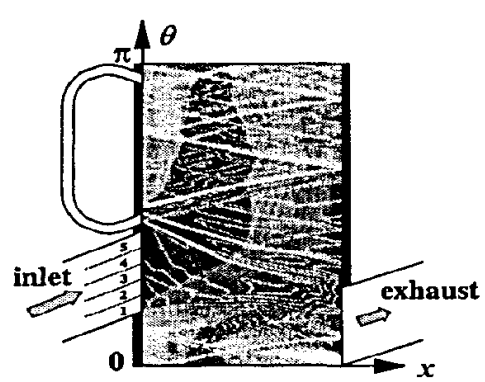

a) Temperature (shades) and pressure (lines)

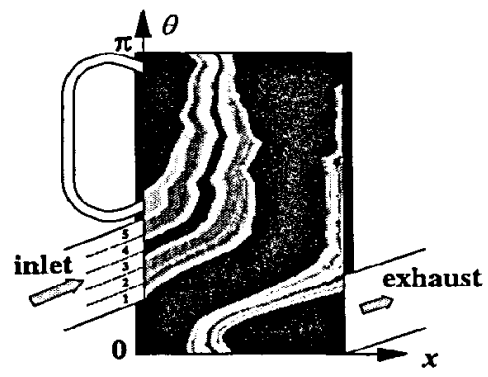

b) Fuel fraction

Fig. 4 Design point simulation contours.

by the reinjected hot gas from the passage fed by leading channels. The center sectors may be left unfueled, as long as fueled sectors are contiguous with ignited sectors, so that the two flame fronts propagating into the charge will reach all flammable mixture. Only five uniform-width sectors are used, partly for computing convenience, whereas an actual design may use more sophisticated stratification. Finer stratification is generally not useful, considering that distortion of interfaces on the scale of the channel width will quickly diffuse the concentration gradient. However, finer stratification may be desired near the ignition sources to better control fuel concentration at ignition, before significant mixing can occur.

In addition to distributing the fuel such that each fueled sector contains a mixture within flammable limits, the equivalence ratio in sector 5 is kept at 0.5 or higher, irrespective of inlet temperature. This gas when combusted supplies the reinjection passage, suffers leakage at the wall, and serves as the residual gas ignition source for the next inlet charge. This condition will help ensure a lit combustor, but may not minimize ignition delay for each fresh charge. It may be necessary to supply a very small quantity of additional fuel to the reinjection passage to attain near stoichiometric conditions in the relatively small mass flow in it (2-5\% of charge mass). This level of design sophistication is beyond the scope of this study.

\section{Results}

Wave diagrams for converged simulations are presented in Figs. 4-6 for the design point, part $(40 \%)$ load, and idle operating conditions, respectively, of the engine. The rotor speed was adjusted to attain correct wave timing, by visual inspection of preliminary wave diagrams. At the converged solutions it ensures no backflow when the inlet is opened, and that the shock wave from the exhaust port arrives just after the inlet closes. It is seen that the wave pattern does not vary qualitatively, although naturally, the highest pressures are generated at the highest load. If the desired speed schedule is nearly proportional to the main shaft speed schedule, the wave rotor could be geared to it; otherwise, an electrically driven or freewheeling rotor is envisaged. In any case, the drive power required will be minimal.

The position of the temperature discontinuities, between hot gas initially in the channel and colder fresh inflow, indicates that approximately half the channel volume is purged and refilled in each cycle at all loads. Note that the apparent slight mismatch between the port positions and gradients in the adjacent gas properties is due to the finite channel width and consequent gradual opening and closing of ports.

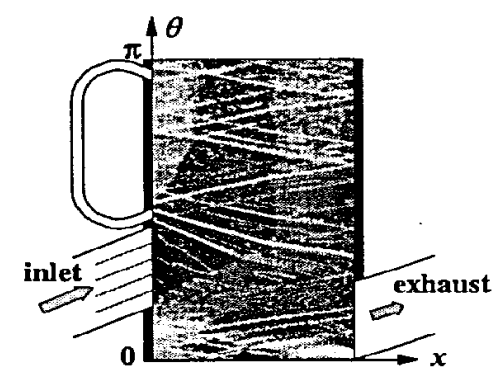

a) Temperature (shades) and pressure (lines)

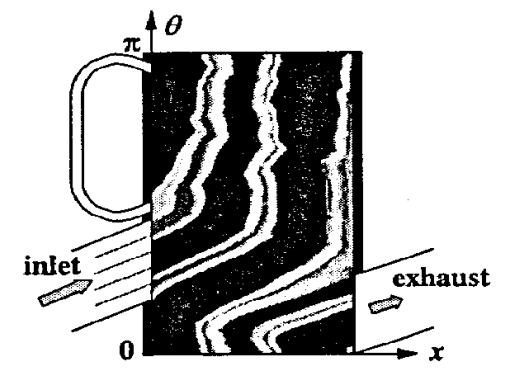

b) Fuel fraction

Fig. 5 Part-load point simulation contours.

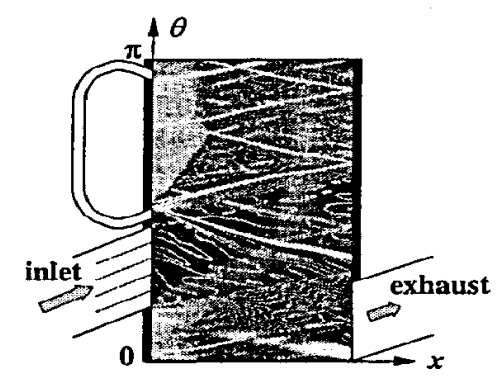

a) Temperature (shades) and pressure (lines)

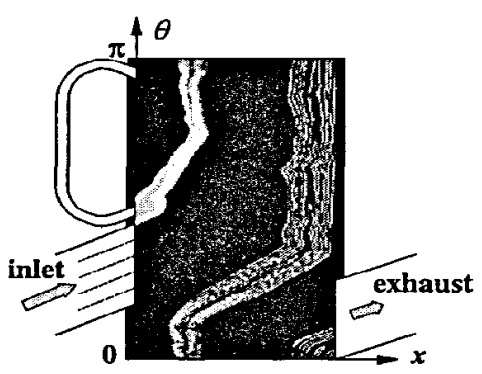

b) Fuel fraction

Fig. 6 Idle point simulation contours.

Quantitative information for the gas flow in the ports is presented in Fig. 7 (inlet and reinjection) and Fig. 8 (exhaust), for each operating point. Note the port duration diagram at the bottom. Profiles of dimensionless velocity, temperature (Kelvin), and unburned fuel are time averaged. Inlet stoichiometry and exhaust fuel concentration are both presented in terms of fuel-air equivalence ratio $\phi$, defined broadly to reflect fuel concentration:

$$
\phi=\left[z_{1} /\left(z_{2}+z_{3}\right)\right]\left(1 / f_{s}\right)
$$

It will be observed that the stratification technique employed here results in a significant temperature gradient in the exhaust with generally higher temperatures in the leading side of the exhaust duct. It is also seen that there is some amount of unburned fuel in the exhaust. The reason for this is that fuel that has diffused into adjacent unfueled air forms a mixture below the flammability limit and will not be consumed. These two observations should be treated with caution as discussed in the next section. 
Table 4 Simulated wave-rotor performance and temperature data

\begin{tabular}{lccc}
\hline \hline Load & Design & $40 \%$ & Idle \\
\hline Speed $N, \mathrm{rpm}$ & 16,800 & 14,400 & 13,200 \\
Wheel mach number $M_{w}$ & 0.29 & 0.27 & 0.28 \\
Mass flow, kg/s & 2.21 & 1.67 & 0.88 \\
Corrected flow $\dot{m}_{c}, \mathrm{~kg} / \mathrm{s}$ & 2.21 & 2.23 & 2.24 \\
Channel combustion efficiency, $\%$ & 91 & 77 & 80 \\
Pressure gain, $\%$ & 23 & 13 & 3 \\
Exhaust stagnation temperature, K & 1,290 & 930 & 630 \\
Maximum turbine inlet temperature. K & 1,360 & 1,060 & 680 \\
Cavity temperature, K & 1,260 & 1,200 & 870 \\
\hline
\end{tabular}
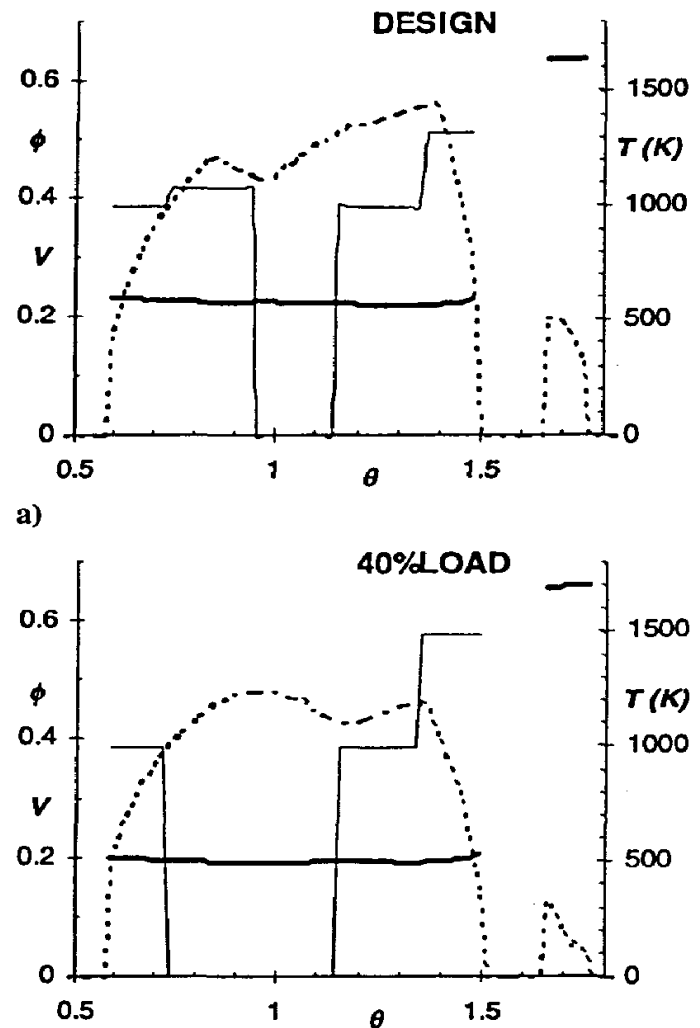

b)

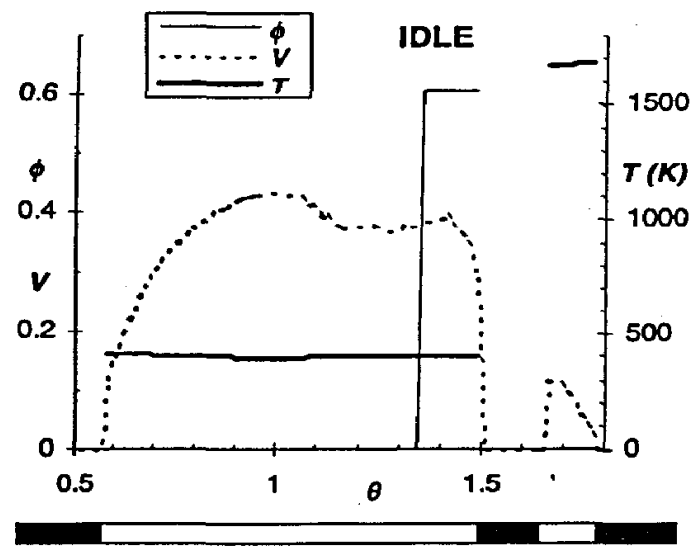

c)

Fig. 7 Inlet and reinjection port profiles for: a) equivalence ratio $\phi$, b) dimensionless velocity $V$, and c) static temperature $T$.

The major performance measures and average exhaust conditions are listed in Table 4. As load changes, the speed $N$ is adjusted for wave timing, and the mass flow automatically adjusts for speed, heat rate, and back pressure. Observe that $N$ falls only about $20 \%$ from full load to idle and that there is no significant change in corrected flow and wheel Mach number, respectively, defined as

$$
\dot{m}_{c}=\dot{m}\left(p^{*} / p_{\mathrm{in}}\right) \sqrt{T_{\mathrm{in}} / T^{*}}
$$

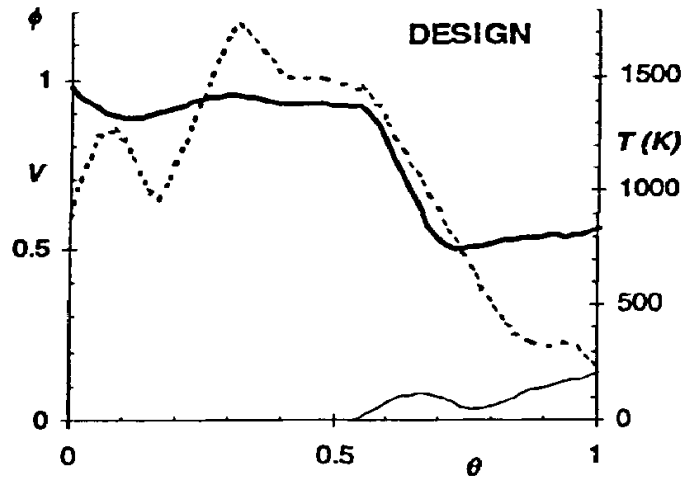

a)

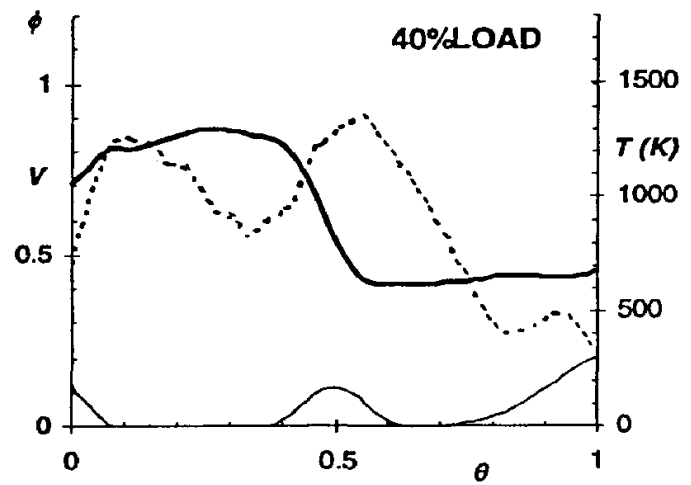

b)

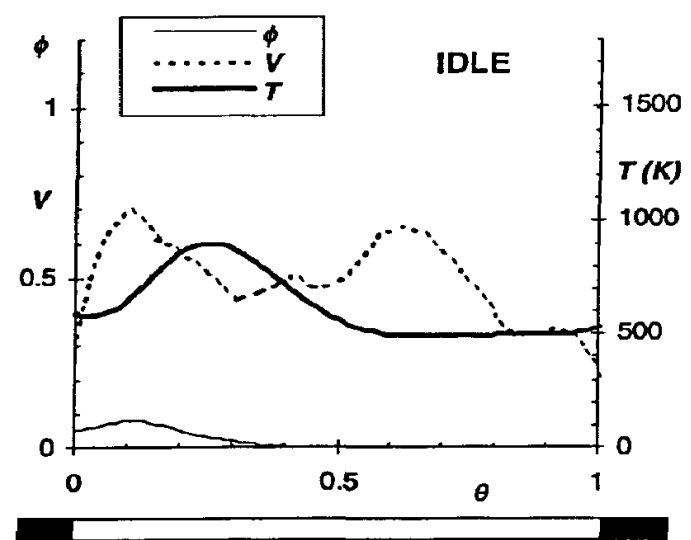

c)

Fig. 8 Exhaust port profiles for: a) equivalence ratio $\phi$, b) dimensionless velocity $V$, and c) static temperature $T$.

and

$$
M_{w}=2 \pi r N / 60 a_{\mathrm{in}}
$$

The maximum turbine inlet temperature is the stagnation temperature if all residual fuel was reacted in the exhaust duct. The fuel rate was adjusted to approximately match the nominal turbine inlet temperature of Table 2 . The pressure gain is penalized by incomplete combustion in the channel, but the design-point penalty does not appear to be significant in comparison to the complete-combustion calculations of Ref. 7.

The computed steady-state rotor wall temperature (assuming no axial conduction in the wall) is plotted in Fig. 9. At the design load, peak wall temperature exceeds the turbine inlet temperature $(1300 \mathrm{~K})$ only in a short segment of the rotor; if the unaccounted longitudinal conduction in the rotor is strong, the temperature everywhere would approach the mean rotor temperature, $1145 \mathrm{~K}$.

The temperatures of the gas adjacent to the end walls are plotted in Figs. 10 and 11, after averaging over fluctuations due to finite channel width. Here it is seen that the inlet side wall remains very hot throughout the load range, nearly $2000 \mathrm{~K}$, except near the inlet 


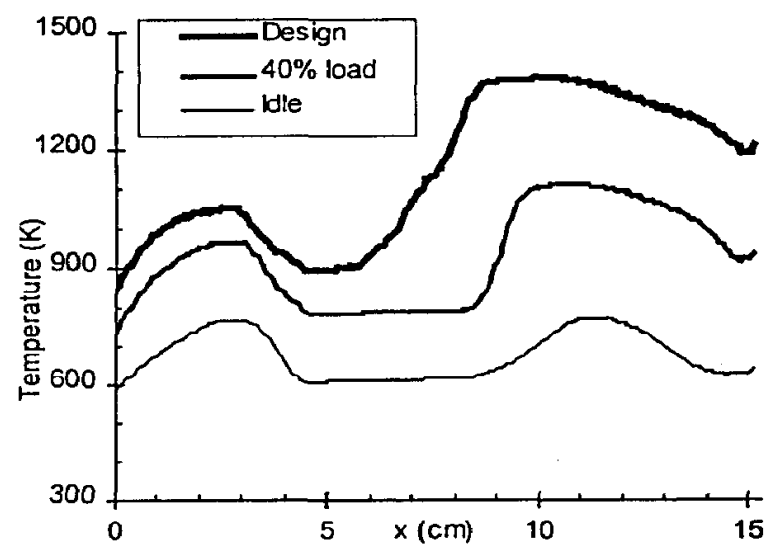

Fig. 9 Rotor wall temperature.

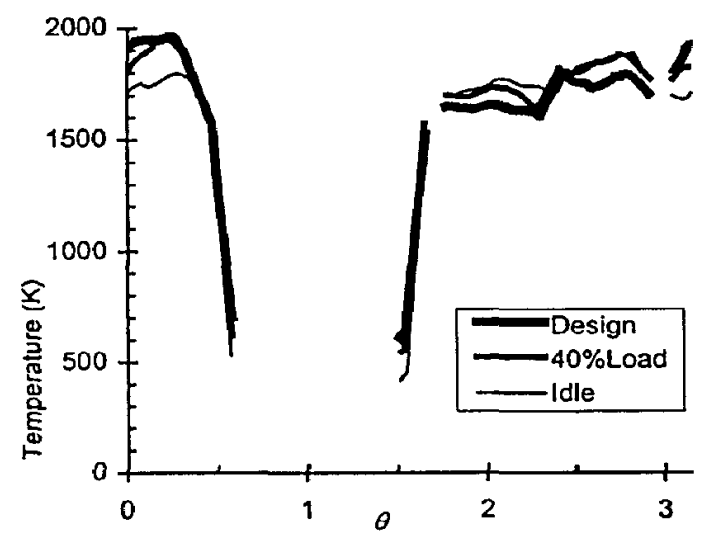

Fig, 10 Inlet-side end wall gas temperature.

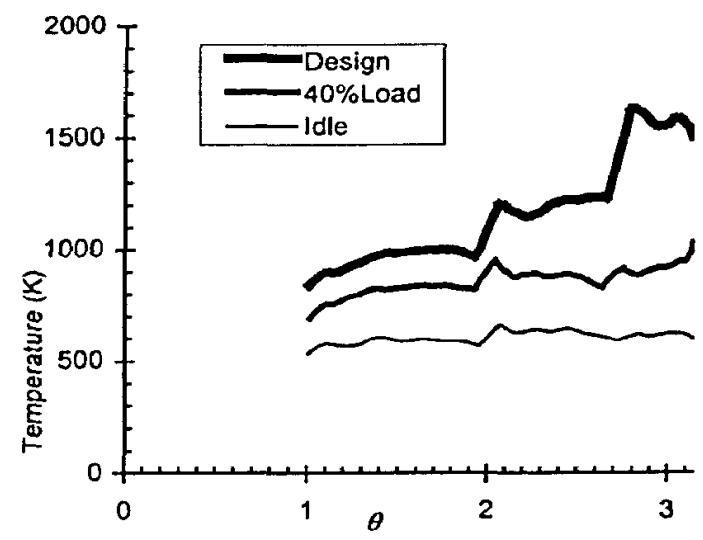

Fig. 11 Exhaust-side end wall gas temperature.

port itself. The exhaust side wall is considerably cooler, especially at the lower loads. This is not surprising because it is a consequence of the stratification strategy and gas particle paths as revealed in the wave diagrams.

\section{Discussion}

The major impact of stratification, necessitated by the imposed flammability limits, is on the exhaust temperature profile and on the rotor and casing temperatures. These distributions of temperature in the device will have major implications for its thermomechanical design and integration with downstream turbomachinery.

The gradients in exhaust temperature are attenuated by diffusion, but peak gas temperatures associated with ignition-zone stoichiometry will be above the design turbine-inlet temperature. The duct joining the wave rotor to the turbine must provide large-scale mixing, possibly driven or aided by the velocity gradients and channelto-channel fluctuations, and at affordable cost in pressure gain. To provide an acceptable radial pattern factor to the turbine, it is desirable to orient remaining temperature variations in the turbine's circumferential direction.

The rotor and end-wall temperature calculations are intended to illustrate the thermal issues related to design of internalcombustion wave rotors. The rotor temperature determined should be treated with caution, as it has been suggested in two-dimensional calculations ${ }^{15}$ that boundary-layer redistribution of fluid may result in temperatures near the wall that differ significantly from onedimensional cell-averaged values. The results for the end walls are probably less disputable and show that these can be extremely hot (up to $2000 \mathrm{~K}$ ). This is particularly so for the inlet side, which provides the recirculation of hot gas for ignition in the wall-adjacent gas. These walls will need dedicated cooling methods and sufficient thickness to minimize warpage. The temperature of the exhaust-side wall depends on the width of the central unfueled sector and could be kept down even at higher loads by more skewed stratification, albeit at the expense of greater exhaust temperature variation. Besides heating the end walls, the high gas temperatures at the leakage gap leads to the high cavity temperatures shown in Table 4 . This could be a serious problem for temperature management of bearings, pressure walls, and other components. It is not feasible to purge this space, as cooler air is not available at a greater pressure than that created by leaking combustion gas.

Although the simulation results appear plausible, it is necessary to preface further discussion of their significance with some caveats about the simulation model. Real combustion fronts are nonplanar and are controlled by phenomena occurring at much smaller scales than can be resolved in these simulations. The use of a single turbulence parameter $\varepsilon_{t}$ to encapsulate all of the complexities of freestream and boundary-layer turbulence, large-scale vortical structures, and subgrid unsteadiness of real flows in a wave rotor is a major weakness of the simple eddy diffusivity model. It is recognized that gradient-diffusion models are truly applicable only to turbulent motions of smaller scale than the gradient zone itself. ${ }^{16}$ The combustion model based on this turbulence model suffers from this limitation as well. Although this is about the best one could hope for with a one-dimensional grid, it necessarily requires circumspect interpretation of the results. If significantly different turbulence levels and flame speeds are attained in a wave rotor, the designer would adjust its rotation speed to match, but would probably not escape this paper's conclusions about thermal loads.

A number of mechanisms exist for generation of large-scale turbulence. Pressure waves interacting with flames or other density gradients or discontinuities produce vorticity by baroclinic forces (Richtmyer-Meshkov instability), and this can create large vortical structures, as borne out by two-dimensional wave rotor flow calculations. ${ }^{15}$ Centripetal and Coriolis forces have a similar effect. Large-scale mixing also occurs when hot gas is reinjected at high speed to penetrate the fresh mixture over a distance greater than the channel width. Yet another mechanism for generation of such structures is the opening and closing of channels of finite width in the presence of significant pressure differences between the port and the channel. The partitions intended for stratification may add to stirring at the inlet plane. It can be shown that turbulence on the scale of the channel width will typically not dissipate significantly over the intake period of a wave cycle. ${ }^{8}$ The fixed eddy diffusivity probably overestimates small-scale mixing at the scale of the computationalcell size, but completely disregards large-scale stirring or redistribution (as distinct from mixing ${ }^{17}$ ). As the large structures break down, their energy reaches scales that beneficially promote mixing late in the cycle and in the exhaust duct.

These observations should imply that the combustion inefficiency associated with local diffusion of fuel to adjacent air may be exaggerated, and any such unreacted fuel will tend to be stirred in with hot gas and burn up. Of course, fuel energy released at duct pressure contributes nothing to pressure gain, but will be available to the turbine. The temperature gradients in the exhaust flow will also be less steep than shown. Nevertheless, the stratification will result in some temperature variation, and it appears from these simulations 
that the cooler gas is always concentrated at the trailing wall of the exhaust duct, where the flow velocity is relatively lower. This consistency might be exploited to extract cooling gas for the duct walls and possibly the turbine components. The availability of cooling air at sufficient pressure is a major issue in any pressure-gain scheme. It is conceived that the hotter gas will be routed through an aerodynamically shaped liner to the turbine guide vanes, and the cooler gas will be directed to the space between the liner and the pressure wall.

The combustion model assumes virtually instantaneous ignition of fresh mixture by adjacent hot gas. This is kinetically plausible, the ignition process being more akin to intermittent pausing and resumption of a propagating flame rather than initiation of a new flame (as would be the case of electric spark ignition and flame kernel growth, a process that involves long ignition delay). The chemical activity and active radical concentration levels of combustion products remains high for many milliseconds, long enough for even relatively slow internal-combustion (IC) engines to exploit for enhanced ignition. ${ }^{18,19}$

The source of any ignition delay or extinction is more likely to be governed by the fluid mechanics of mixing, rather than chemistry. Excessive mixing due to freestream and inlet-generated turbulence may defeat the intent of stratification and create over-lean zones that will not react. Overzealous design of the reinjection of hot gas at too high a velocity could retard ignition due to high initial strain rates. ${ }^{20}$ Thus, the hot gas recirculation passage design must be optimized to procure the gas at the right pressure for fastest reignition and combustion. It may turn out that the most effective recirculation of combustion products is from immediately preceding passages by the use of a pocket in the end wall, rather than by a duct from the highest-pressure passages. Optimally, the Damkohler number (ratio of chemical to mixing timescales) must be near unity.

A likely consequence of inconsistent ignition and combustion is cycle-to-cycle variability, and the stability issues discussed in Ref. 8 should be considered. To avoid the difficulties associated with hot gas leakage and reinjection, alternate ignition methods should be investigated. These may include laser ignition, or ignition by rotorbased spark electrodes, which can initiate combustion in the interior of the channel rather than at the ends.

\section{Conclusions}

A computational method for combustion in wave rotors has been further developed to allow rapid simulation of stratified premixed turbulent combustion. Flame propagation is simulated by the use of a mixing-controlled combustion model, based on fixed eddy diffusivity. Multiple species and reaction steps are accommodated, and simple ignition-temperature and mixture-flammability limits are incorporated. Although the flow-loss models used are based on validated studies, the combustion model awaits calibration by experiments or more detailed multi-dimensional calculations. Using plausible model parameters, including a flammability limit, simulations of combustion and wave-rotor flows are presented for engine operating points covering the full range of normal operation of a waverotor combustor for a small turbine engine with nominal pressure ratio of about 8 and turbine inlet temperature of $1300 \mathrm{~K}$. Although lacking the ability to resolve important flow features that may influence mixing and combustion, these one-dimensional simulations provide a general illustration of the issues facing IC wave-rotor design.

The goal of achieving high overall combustion rates within a small rotor appears feasible if fuel distribution is properly designed and actively controlled over the load range. A strategy for fuelair mixture stratification is developed that appears to satisfy the requirements for cycle-to-cycle reignition and adequate combustion rates and efficiency. Fuel is concentrated at the leading and trailing edges of the inlet port under load, and only at the trailing edge at idle. As load is varied, the driven speed of the rotor is adjusted over a narrow range, so that the cyclic wave pattern in the channel remains qualitatively similar. The assumption of a uniform fixed eddy diffusivity operating on the scale of the computational grid ignores the large-scale stirring mechanisms known to exist in wave rotors, which may modify the required stratification and the resulting flow and combustion processes.

The requirement for nonuniform fuel distribution leads to temperature profiles in the gas driving the turbine and also affects the rotor temperature distribution and the end wall temperatures. Excessive local temperatures are generated that can negatively impact the rotor, housing, and downstream turbine. Peak rotor temperature will be comparable to the turbine inlet temperature, implying that similar materials could be used for construction of the turbine and wave rotor if each is similarly cooled or uncooled.

Of equal or greater concern are the high temperatures of the gas near the end walls, which result in hot end walls (up to $2000 \mathrm{~K}$ if uncooled) and leakage of hot gas to the housing cavity. The cavity will reach $1260 \mathrm{~K}$ and 12.7 atm at full load, tending to overheat sensitive areas, including the pressure walls and rotor bearings. There is normally no source of fluid for purging and cooling the space at these conditions. Warping of the end walls due to temperature nonuniformity will make leakage particularly difficult to control. Management of the end-wall temperature in particular, and thermal management of all other components of the system, appear to be critical to the feasibility of this concept. Implications for the weight and durability of the rotating combustor, with its large pressure fluctuations, and of the entire system, must be explored further.

\section{Acknowledgments}

The author is grateful for the valuable assistance of Daniel Paxson of the NASA John H. Glenn Research Center at Lewis Field, who developed the original wave rotor code on which this work is based and helped create the figures in this paper.

\section{References}

'Welch, G. E., Jones, S. M., and Paxson, D. E., "Wave Rotor-Enhanced Gas Turbine Engines," Journal of Engineering for Gas Turbines and Power, Vol. 119, No. 2, April 1997, p. 469; also AIAA Paper 95-2799, July 1995; also NASA TM-106998, 1995; also Army Research Lab.-TR-806, 1995.

${ }^{2}$ Nalim, M. R., "Wave Cycle Design for Wave Rotor Engines with Limited Nitrogen Oxide Emissions," Ph.D. Dissertation, Sibley School of Mechanical and Aerospace Engineering, Cornell Univ., Ithaca, NY, Jan. 1994.

${ }^{3}$ Shreeve, R. P., and Mathur, A., (eds.), Proceedings of the 1985 ONR/ NAVAIR Wave Rotor Research and Technology Workshop, Naval Postgraduate School, Monterey, CA, NPS-67-85-008, May 1985.

${ }^{4}$ Nalim, M. R., "Thermodynamic Limits of Pressure Gain and Work Production in Combustion and Evaporation Processes," AIAA Paper 98-3398, July 1998.

Jones, S. M., and Welch, G. E., "Performance Benefits for Wave RotorTopped Gas Turbine Engines," American Society of Mechanical Engineers, Paper 96-GT-075, June 1996; also NASA TM-107193, 1996.

${ }^{6}$ Wilson, J., and Paxson, D. E., "Wave Rotor Optimization for Gas Turbine Engine Topping Cycles," Journal of Propulsion and Power, Vol. 12, No. 4, 1996, pp. 778-785; also NASA TM-106951, 1995.

${ }^{7}$ Nalim, M. R., and Paxson, D. E., “A Numerical Investigation of Premixed Combustion in Wave Rotors," Journal of Engineering for Gas Turbines and Power, Vol. 119, July 1997, p. 668; also NASA TM-107242, 1996.

${ }^{8} \mathrm{Nalim}, \mathrm{M}$. R., "Preliminary Assessment of Combustion Modes for Internal Combustion Wave Rotors," AIAA Paper 95-2801, July 1995; also NASA TM-107000, 1995.

${ }^{9}$ Paxson, D. E., "Comparison Between Numerically Modeled and Experimentally Determined Wave-Rotor Loss Mechanisms," Journal of Propulsion and Power, Vol. 11, No. 5, 1995, pp. 908-914; also NASA TM-106279, 1993.

${ }^{10}$ Strehlow, R. E., Combustion Fundamentals, McGraw-Hill, NY, 1984, pp. $370-380$.

"Chen, Z. H., and Sohrab, S. H., "Flammability Limit and LimitTemperature of Counterflow Lean Methane-Air Flames," Combustion and Flame, Vol. 102, Nos. 1/2, 1995, pp. 193-199.

12 Magnussen, B. F., and Hjertager, B. H., "On Mathematical Modeling of Turbulent Combustion with Special Emphasis on Soot Formation and Combustion," I6th Symposium (International) on Combustion, Combustion Inst., Pittsburgh, PA, 1976, pp. 719-729.

${ }^{13}$ Roe, P. L., "Characteristic Based Schemes for the Euler Equations," Annual Review of Fluid Mechanics, Vol. 18, 1986, pp. 337-365. 
${ }^{14}$ Snyder, P. H., and Fish, R. E., "Assessment of a Wave Rotor Topped Demonstrator Gas Turbine Engine Concept," American Society of Mechanical Engineers, Paper 96-GT-041, June 1996.

${ }^{15}$ Welch, G. E., "Two-Dimensional Computational Model for Wave Rotor Flow Dynamics," American Society of Mechanical Engineers, Paper 96-GT550, 1996; also NASA TM-107192, 1996; also Army Research Lab. TR-924, 1996.

${ }^{16}$ Broadwell, J. E., and Breidenthal, R. E., "A Simple Model of Mixing and Chemical Reaction in a Turbulent Shear Layer," Journal of Fluid Mechanics, Vol. 125, 1982, p. 397.

${ }^{17}$ Broadwell, J. E., and Mungal, M. G., "Large-Scale Structures and
Molecular Mixing," Physics of Fluids A, Vol. 3, No. 5, 1991, p. 1193.

${ }^{18}$ Gussak, L. A., "High Chemical Activity of Incomplete Combustion Products and a Method of Prechamber Torch Ignition for Avalanche Activation of Combustion in Internal Combustion Engines," Society of Automotive Engineers, Paper 750890, Oct. 1975.

${ }^{19}$ Lu, J., Gupta, A. K., Pouring, A. A., and Keating, E. L., “A Preliminary Study of Chemically Enhanced Autoignition in an Internal Combustion Engine," Society of Automotive Engineers, Paper 940758, Feb. 1994.

${ }^{20}$ Cattolica, R., and Vosen, S., "Combustion Torch Ignition: Fluorescence Imaging of $\mathrm{OH}$ Concentration," Combustion and Flame, Vol. 68, No. 3, 1987, pp. 267-281.

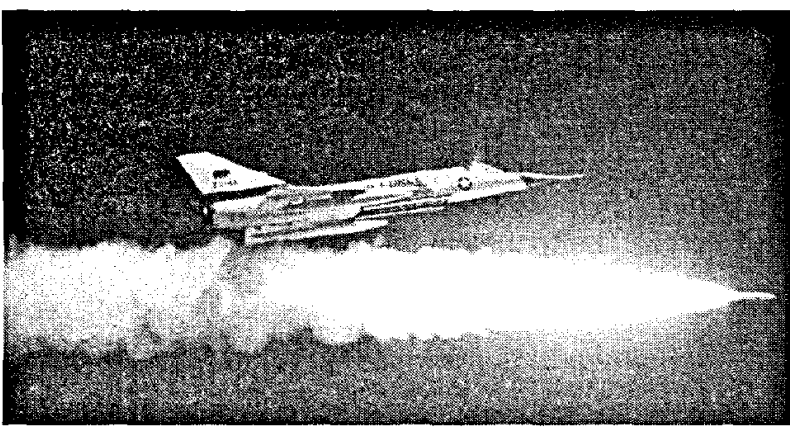

G.E. Jensen, United Technologies Corporation, and David W. Netzer, Naval Postgraduate School, editors

1996, $650 \mathrm{pp}$, Hardcover

ISBN $t-56347-118-3$

List Price: $\$ 104.95$

AIAA Member Price: $\$ \mathbf{8 9 . 9 5}$

\section{Tactical Missile Propulsion}

$\mathrm{W}$ ith contributions from the leading researchers and scientists in the field, this volume is a compendium of advances in tactical missile propulsion. The book provides today's designers with practical solutions in their design and selection considerations. Design of solid rocket cases, nozzles, motors, liquid rockets, gas turbines, ramjets, and high Mach number applications are covered.

\section{Contents:}

Introduction - Design Concepts and Propulsion Definition Liquid Rockets • Solid Rocket Motor Design - Solid Propellant Grain Structural Design and Service Life Analysis • Solid Rocket Nozzle Design - Solid Rocket Case Design • Solid Rocket Plumes • Insensitive Munitions for Solid Rockets

- Gas Turbines • Liquid Fueled Ramjets • Ducted Rockets • Solid Fueled Ramjets • High Mach Number Applications

\section{OAIAM}

American Institute of Aeronautics and Astronautics Publications Customer Service 9 Jay Gould Cl., P.O. Box 753, Waldort, MD 20604 Fax 301/843-0159 Phone 800/682-2422 E-mail aiaa@tasco1.com 8 am-5 pm Eastern Standard Time
$C A$ and VA residents add applicable sales tax. For shipping and handting add $\$ 4.75$ for 1-4 books (call for rates for higher quan pities). All individual orders-including U.S., Canadian, and foreign - must be prcoaid by personal or campany check, traveler's heck interion pack, in to payable to AIAA in U.S dollars, drawn on a U.S. bank Orders from Ibranies, corporalions, governiment agencies, and university and college bookstores must be accompanied by an authorized purct tase order. All other bookstore orders must be prepaid. Please allow 4 weeks for delivery. Prices are subject to change without notice. Returns in sellable condition will be accepted within 30 days. Sormy we cannot accept returns of case studies, conterence proceedings, sale items, or sottware (unless defective). Non-U.S. residents are
responsible for payment of any taxes required by their government. 